Research Article

\title{
IDBR: Iot Enabled Depth Base Routing Method for Underwater Wireless Sensor Network
}

\author{
Umer Farooq, ${ }^{1}$ Mohib Ullah $\mathbb{D}^{1},{ }^{1}$ Rafi Ullah Khan $\mathbb{D}^{1},{ }^{1}$ Abdullah Alharbi, ${ }^{2}$ \\ Muhammad Irfan Uddin $\left(\mathbb{D},{ }^{3}\right.$ Muhammad Inam Ul Haq, ${ }^{4}$ and Wael Alosaimi ${ }^{2}$ \\ ${ }^{1}$ Institute of Computer Science and Information Technology, The University of Agriculture, Peshawar, Pakistan \\ ${ }^{2}$ Department of Information Technology, College of Computers and Information Technology, Taif University, P.O.Box 11099, \\ Taif 21944, Saudi Arabia \\ ${ }^{3}$ Institute of Computing, Kohat University of Science and Technology, Kohat 26000, Pakistan \\ ${ }^{4}$ Department of Computer Science and Bioinformatics, Khushal Khan Khattak University, Karak, Pakistan
}

Correspondence should be addressed to Rafi Ullah Khan; rafiyz@gmail.com

Received 29 August 2021; Accepted 17 September 2021; Published 8 October 2021

Academic Editor: Guolong Shi

Copyright (C) 2021 Umer Farooq et al. This is an open access article distributed under the Creative Commons Attribution License, which permits unrestricted use, distribution, and reproduction in any medium, provided the original work is properly cited.

\begin{abstract}
Underwater wireless sensor networks (UWSNs) gained the attention of researchers due to their substantial applications in various fields. The major application areas of UWSN are environmental monitoring, underwater oil and gas extraction and military surveillance, smart farming, communication, and others. However, UWSNs are also prone to significant issues, such as limited network lifetime, the low processing capability of nodes, high energy consumption to run routing protocols, and difficult node replacement. Therefore, enhancing the lifetime of UWSN by reducing energy consumption and processing is a research issue. In this research, we proposed the IoT enabled depth base routing method (IDBR) to utilize energy efficiently. The performance of the proposed IDBR method is compared with conventional DBR protocol using simulation conducting in MATLAB. The performance of both the methods (i.e., IDBR and DBR) is evaluated in network energy consumption, the number of alive nodes, sink utilization, and end-to-end delay. The simulation-based experiment results show that IDBR consumes $27.7 \%$ less energy and increases network stability than the DBR. Similarly, the utilization of the surface sinks in IDBR is more as compared to DBR as in IDBR, and sinks work as relay forward data to the base station without processing which increases the power of field nodes. The proposed mechanism improves the network's lifetime and increases the accessibility and security of the sensed data.
\end{abstract}

\section{Introduction}

The two-third part of planet Earth consists of water in various forms such as oceans, lakes, streams, rivers, and glaciers $[1,2]$, containing an ample quantity of valuable resources. This shows the importance of exploring the underwater medium. Exploring underwater medium is also essential for monitoring pollution [3], military application [4], oil rig maintenance [5], marine life and environmental monitoring [6], etc. The recent advancement in underwater sensors technology has led to the possibility of underwater explorations using sensors effectively [7]. UWSN combines autonomous sensors designed to sense the pressure, temperature, water quality, and other water-related properties [8].
The data gathered by the sensors is then sent to the sink node for analysis through other sensor nodes using acoustic waves. The significant problems related to the UWSNs are limited network lifetime, the low processing capability of nodes, and high energy consumption to run routing protocols [2]. As the battery replacement in UWSN is a challenging task, an energy-efficient routing method can be useful to improve the lifetime of the network.

Internet of Things (IoT) is a network concept between physical objects embedded with software, sensors, and other communication hardwares $[9,10]$. IoT's primary purpose is to provide efficient communication and data collection for the devices used for various tasks such as traffic monitoring, data collection device usage, monitoring and maintenance, 
smart cities, and smart agriculture, [11]. IoT systems have four major components: sensors or nodes, a communication module, a data processing module, and a user interface [12]. Sensors are used to collect the data; a communication module is used to send the data to the cloud, and the processing module is used to process the data, while the user interface is used to report the user about the situation [13].

Depth-based routing (DBR) protocol is a popular UWSN routing method used to find the shortest path between the data sending node and sink node [14]. However, it only focuses on the depth of the sensor node to decide while ignoring the other parameters such as storing and processing capabilities of sink nodes. The significant problems related to the UWSN are high energy consumption and limited network life. A routing protocol with efficient but limited routing protocol steps may deal with the problems mentioned above [15]. To enhance the lifetime of the UWSN, it is essential that the processing at the sink node and the base station's data storage should be reduced. This solution is possible if IoT characteristics are enabled in the architecture of UWSN.

In this research, we proposed a new method, which will incorporate IoT features with UWSN. The IoT enabled smart sinks will only forward data to the base station instead of processing or storage. It will also deliver the sensed data to the nearest sink for relaying data to the base station when the power of the sink node becomes low during communication. The proposed scheme will use the cloud for data storage and data processing with IoT services. The data collection will be the responsibility of sinks in underwater networks, whereas storage and processing capabilities will be the responsibility of the cloud. The proposed mechanism improves the network's lifetime and increases the accessibility and security of the sensed data.

The rest of the paper is organized as follow: In Section 2, we discussed the literature review. Sections 3 and 4 discuss our proposed IDBR routing method and the methodology to conduct experiments, respectively. The results and conclusion of the experiments are discussed in Sections 5 and 6 , respectively.

\section{Literature Review}

The battery lifetime of sensor nodes in (UWSN) is limited. Therefore, some of the underwater sensor nodes disappeared from the UWSN during data transmission. Ammar et al. proposed a MAC-based protocol that uses depth adjustment and splitting mechanism based on the greedy approach [16]. Their proposed protocol solved the problem of isolated nodes and thus improved the lifetime of the network. Similarly, Wang et al. presented a new contention-based energyefficient Media Access Control (MAC) for underwater sensor networks [17]. They combined the postponed access technique of CSMA/CA and multiplexing-based Spatial Division Multiple Access (SDMA) to get the real-time transmission of data with high speed. The proposed technique ensures the idle state of the channel first by performing an acoustic handshake to get the sensor node location information. If not, it waits for the next time slot and executes post-

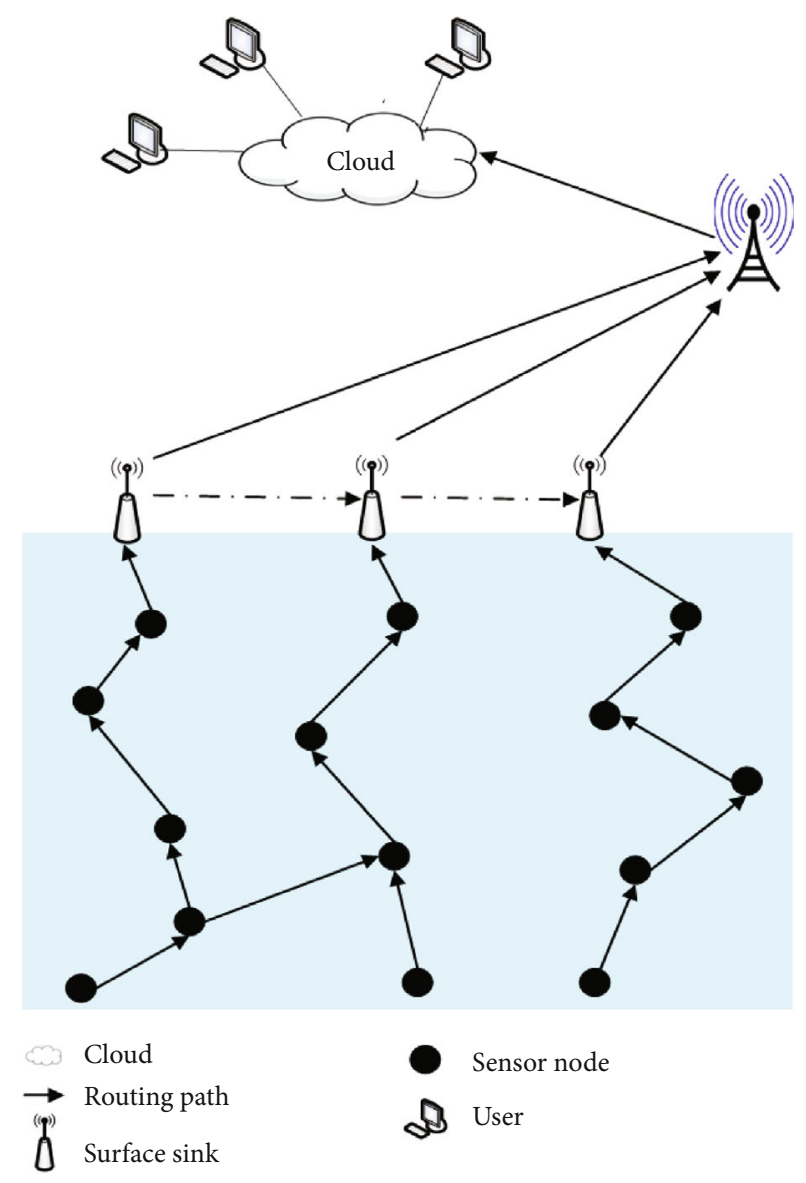

FIgURE 1: Proposed IDBR: IoT enabled depth base routing method.

poned access to hold the channel again. So, for detecting whether the channel is ready for the optical transmission, an optical handshake is used, and at the same time, beam arrangement is performed. In the end, by using optical communication, the sensor nodes finally transmit data in the network. If the channel condition does not satisfy the optical communication requirements, then the small amount of data packets is transmitted using acoustic communication with very high precedence. The results showed that the proposed protocol doubled throughput, and nodes' lifetime was $30 \%$ longer than pure acoustic communication and reduced network operation.

Khan and Dwivedi proposed LRCLE, a node location finding algorithm to reduce the computational cost and minimize the node location error [18]. LRCLE uses the Global Positioning System (GPS) and temperature to find the node's location with a minimum error margin. The proposed algorithm LRCLE improved in various parameters like communication cost, localization coverage, and localization error compared to other known algorithms. While Ali et al. proposed a timebased reliable link (TBRL), the novel routing scheme for IoT enabled underwater sensor networks [19]. TBRL is categorized into three different phases. In the initial phase, the proposed scheme discovered all sensor nodes topology innetwork with the help of the topology algorithm. In the next phase, each established link reliability has been calculated using the model 


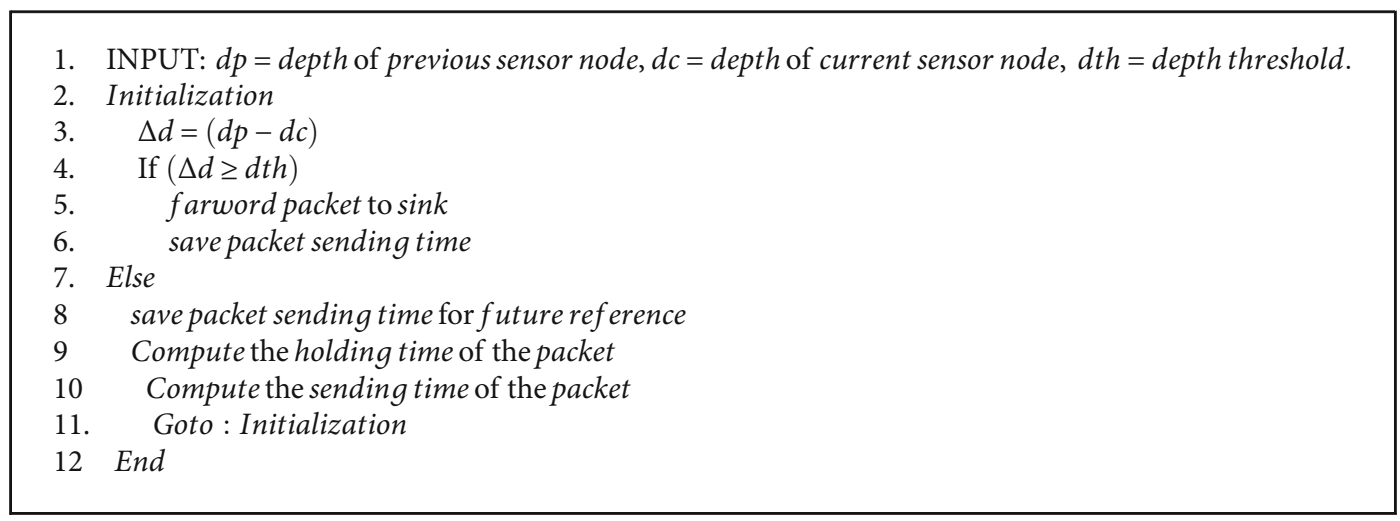

Algorithm 1: Packet forwarding algorithm for sensor node.

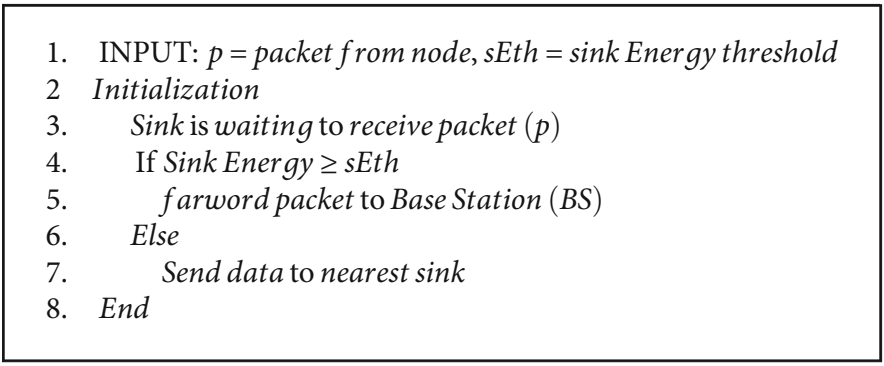

Algorithm 2: Packet forwarding algorithm for sink node.

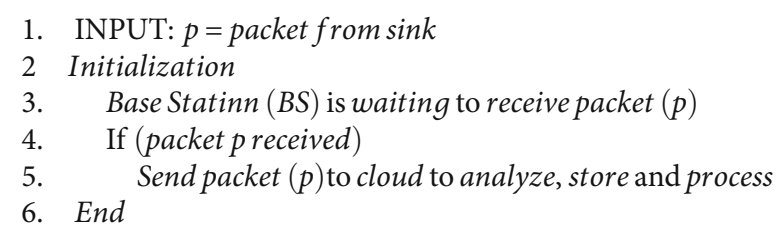

Algorithm 3: Packet forwarding algorithm for Base Station.

of two sensor nodes reliable for a smart environment. In the third phase, all the possible paths are computed and selected the most reliable path for sending the data packet. The TBRL compared with Depth-Based Routing (DBR) and reliable energy efficient routing method in order to check the consumption of energy, end-to-end delay, and packet delivery ratio of IoT enabled underwater sensor network.

Usman et al. concentrated on IoT-UWSNs and supported smart city vision, based on terrestrial Away Cluster Head with Adaptive Clustering Habit $(\mathrm{ACH})^{2}$ that was observed in three identified dimensional areas in the underwater environment [1]. The researchers considered three cases: a single sink node on the water's surface, multiple sink nodes on the water's surface, and sink nodes both at and under the water. The underwater $(\mathrm{ACH})^{2}$ $\left(\mathrm{U}-(\mathrm{ACH})^{2}\right)$ is calculated in each situation. In the proposed scheme, they used depth in their proposed U$(\mathrm{ACH})^{2}$ to examine the $(\mathrm{ACH})^{2}$ enactment in the underwater environment.

Menon et al. proposed SEEORVA, an energy-efficient routing protocol for underwater acoustic sensor networks that uses opportunistic routing strategy lightweight encryption method for secure and reliable data transmission [20]. Their proposed method prioritizes the nodes having residual energy above the defined threshold value for data forwarding and thus increasing the network's lifetime. Similarly, Goswami et al. proposed artificial intelligence and neural network-based energy efficient WSN routing protocol for the Intelligent Transport System [21]. They used a hybrid approach of Self-organizing map (SOM) and distributed artificial intelligence (DAI) technique that improves the overall network's lifetime. In contrast, Verma et al. surveyed Hierarchical-based routing protocols for WSN in terms of energy efficiency [22].

\section{IDBR: IoT Enabled Depth Base Routing Method}

DBR is one of the popular UWSN routing protocols that use depth information of the node to make routing decisions [14]. In usual DBR scenarios, sink nodes are situated at the water surface in a lifebuoy, and based on the depth information of every sensor node, DBR forwards data packets using a greedy approach. Every data, packet keeps the depth information of its recent forwarder, which is updated at every hop. When a sensor node receives any data packet, it compares the depth embedded in a packet with its depth. If its depth is smaller, it forwards the packet to the next hop, otherwise, drop it. DBR takes data forwarding decisions based on the node's depth information while ignoring the sink nodes' other parameters such as storing and processing 


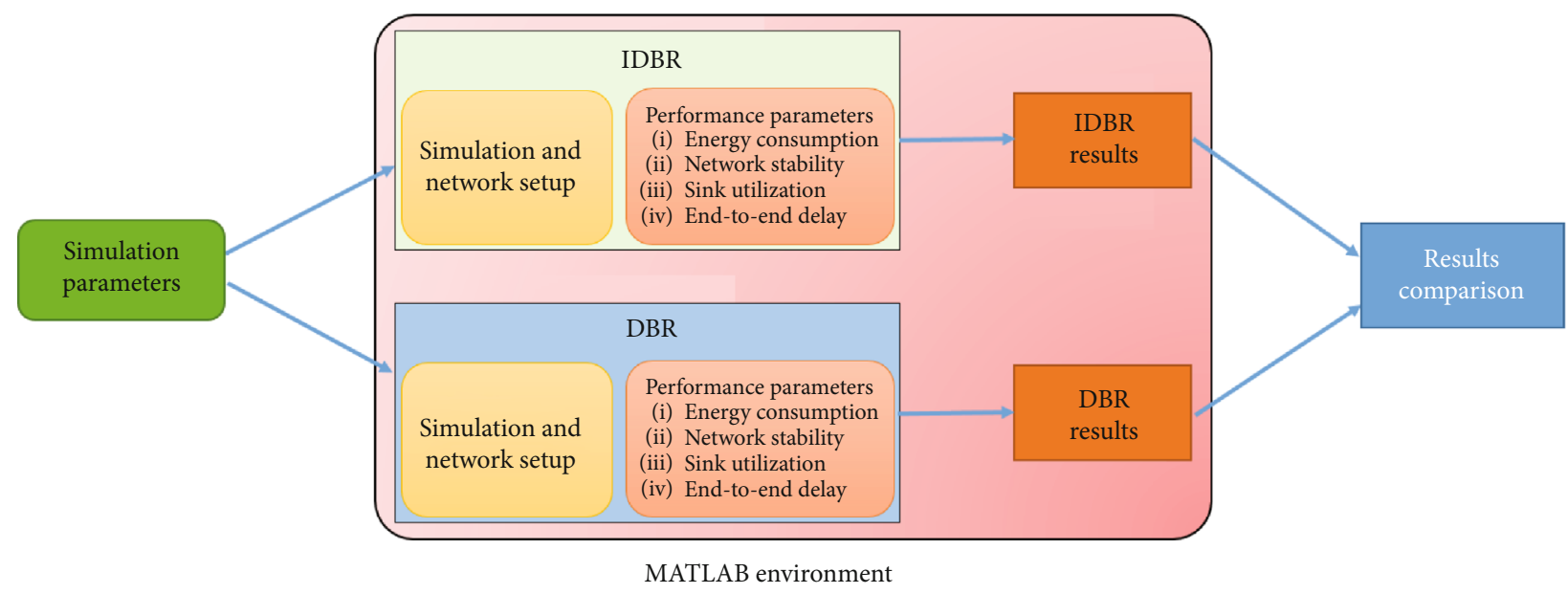

Figure 2: Flow diagram of the proposed methodology.

TABLE 1: Simulation parameters.

\begin{tabular}{lc}
\hline Parameters & Values \\
\hline Simulation tool & MATLAB \\
Size of network & $500 \mathrm{~m} \times 500 \mathrm{~m} \times 500 \mathrm{~m}$ \\
Number of sensor nodes & 225 \\
Initial energy of sensor nodes & $2 \mathrm{~J}$ \\
Range of transmission & $250 \mathrm{~m}$ \\
Number of surface sink nodes & 5 \\
Size of packet & 50 bytes \\
Time of simulation & 8000 seconds \\
Protocols & IDBR (proposed), DBR \\
\hline
\end{tabular}

capability; although, considering other parameters to make routing decisions may improve the routing decisions. However, this may also introduce noticeable computational costs and extra memory.

In our proposed IoT enabled depth base routing method (IDBR), we introduced IoT enabled intelligent sink nodes. The duty of these sink nodes will be to forward the database station instead of storing and processing the data. It will also deliver the sensed data to the nearest sink for relaying data to the base station when the power of the sink node becomes low during communication. The proposed scheme will use the cloud for data storage and data processing with IoT services. In this section, we discuss the working and algorithm of the proposed IBDR method.

3.1. Working of IDBR. The proposed IDBR routing method needed sensor nodes deployed in the field, and IoT enables surface sink node (deployed on lifebuoy), base station, and a cloud. Field sensors are used to collect data from the field. IoT enables surface sink nodes to collect data from the field nodes and sends them to the base station. The base station connects the sinks nodes with the cloud, where data is stored and processed and then sent to the user. The working of the proposed IBDR method is illustrated in Figure 1.
Algorithm 1 is used as a packet forwarding algorithm for the sensor node. When a sensor node receives data in the initial step, it checks whether it is qualified to forward the packet. At this stage, the current node will get information about the depth of the previous node (dp) and its depth $(\mathrm{dc})$. After getting the values of $(\mathrm{dp})$ and ( $\mathrm{dc}$ ), the node will compute the difference between the previous node and current node depth difference $\Delta d=(\mathrm{dp}-$ dc). $\Delta d$ is called holding time computation. Every sensor node uses this holding time to arrange a schedule for packet forwarding in UWSN. If $\Delta d$ is greater than the threshold value $(d$ th $)$, it means that the receiving node is suitable for forwarding packets; otherwise, the node will compute the holding and sending time of the packet and check $\Delta d$ with $d$ th for forwarding the packet. Then, the qualified node will get the previous sending time of the packet for computation and forward it to the sink node. In the case of many packets received for forwarding, the node makes a queue of packets with a FIFO (First In First Out) strategy to entertain the receiving packet.

Algorithm 2 is used as a packet forwarding algorithm for the sink node. Initially, the sink node waits for receiving the packet from the sensor node. After receiving the data, the sink node will check its residual energy against the threshold value sEth. If the value of the residual energy of the sink is greater than or equal to the threshold value, the sink node will send the data to the base station B.S.; otherwise, it will send the data to the nearest sink.

Algorithm 3 is used as a packet forwarding algorithm for the base station. Initially, the base station waits for receiving the packet from the sink node. After receiving the data from the sink node, the base station will send the data to the cloud for storing, processing, and analysis.

\section{Methodology}

For testing the performance of the proposed IDBR routing method against the DBR, we implement both the DBR and IDBR routing methods in MATLAB. The network size for simulation is taken as $500 \mathrm{~m} \times 500 \mathrm{~m} \times 500 \mathrm{~m}$ in which 225 
TABLE 2: Energy consumption.

\begin{tabular}{lccccccccc}
\hline \multirow{2}{*}{ Routing method } & \multicolumn{4}{c}{ Time (sec) } & \multicolumn{3}{c}{ Remaining energy } \\
& 1000 & 2000 & 3000 & 4000 & 5000 & 6000 & 7000 & 8000 & $33.9 \%$ \\
UWSN (DBR) & $10.3 \%$ & $20.2 \%$ & $29.2 \%$ & $37.4 \%$ & $45.5 \%$ & $52.3 \%$ & $59.1 \%$ & $66.1 \%$ & $38.4 \%$ \\
UWSN-IoT (IDBR) (proposed) & $6.6 \%$ & $13.1 \%$ & $18.3 \%$ & $23.2 \%$ & $27.4 \%$ & $31.4 \%$ & $35.2 \%$ & $38.6 \%$ \\
\hline
\end{tabular}

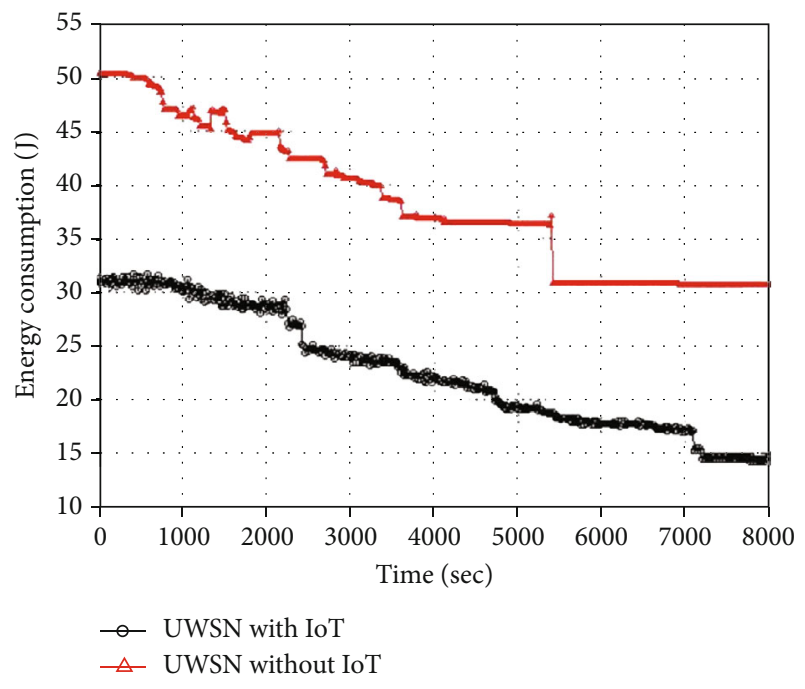

FIGURE 3: Energy consumption.

sensor nodes deploy randomly, with the initial state energy of the sensor node as 2 joules. Five sinks nodes were deployed on the surface of the water for communication with the base station. The simulations of both IDBR and DBR protocols are conducted using the same simulation parameters and network setup. The performance of the proposed IDBR method and DBR is evaluated in terms of energy consumption, the number of alive nodes, sink utilization, and end-to-end delay. After conducting the experiments, the results of both IDBR and DBR are compared. The flow diagram of the proposed methodology is illustrated in Figure 2. The details of the simulation parameters are illustrated in Table 1.

\section{Results and Discussions}

In this research, we proposed IoT enabled IDBR, an energyefficient routing method for UWSN. The performance of the proposed IDBR method is compared with conventional DBR protocol using simulation conducting in MATLAB. The performance of both the methods (i.e. IDBR and DBR) is evaluated in network energy consumption, the number of alive nodes, sink utilization, and end-to-end delay. All experiments were conducted on a workstation with a $4.1 \mathrm{GHz}$ Core i7 processor and 16 GB RAM. This section contains a discussion about the results of the experiments.

5.1. Energy Consumption. Table 2 and Figure 3 show the energy consumption comparison between UWSN without IoT (DBR) and UWSN with IoT (IDBR) calculated at different periods. The results showed that, after 1000 seconds,
DBR utilized 10.3\% energy while IDBR utilized 6.6\% energy. After 5000 seconds, DBR utilized $45.5 \%$ energy while IDBR utilized $27.34 \%$ energy. Similarly, DBR consumed $66.1 \%$ of the total network energy at the end of the simulation, while IDBR consumed $38.4 \%$ of the total network energy. According to the results, the utilization of energy between the sensor nodes during the communication is much more efficient (27.7\% less than DBR) in the proposed method because IoT makes the data forwarding better during the communication, increasing the lifetime UWSN compared to the existing method.

5.2. Network Stability. In the case of network stability, the nodes in the IDBR routing method are more stable than those in the DBR method. Table 3 and Figure 4 show the number of alive nodes compared between UWSN without IoT (DBR) and UWSN with IoT (IDBR) calculated at different periods. The results showed that, after 1000 seconds, the number of alive nodes in DBR was 192, whereas, in IDBR, the number of alive nodes was 212. Similarly, after 5000 seconds, the number of alive nodes in DBR was 131, while in IDBR, the number of alive nodes is recorded as 145 . At the end of the simulation, the number of alive nodes in DBR is recorded as 104, while in IDBR, it is recorded as 108 . According to the results, the network of IDBR is more stable as compared to the DBR.

5.3. Surface Sink Node Utilization. Table 4 and Figure 5 compare surface sink node utilization between UWSN without IoT (DBR) and UWSN with IoT (IDBR) calculated at different periods in terms of power. According to the results, at 1000 seconds, the average sink node utilization in DBR is $121.3 \mathrm{~dB}$, while the average sink node utilization in IDBR is $124 \mathrm{~dB}$. Similarly, at 5000 seconds, the average sink node utilization in DBR is $106.1 \mathrm{~dB}$, while the average sink node utilization in IDBR is $117.6 \mathrm{~dB}$. At the end of the simulation, the average sink node utilization in DBR is $82.02 \mathrm{~dB}$, while the average sink node utilization in IDBR is $100.7 \mathrm{~dB}$. The results showed that the utilization of the surface sinks in IDBR is more as compared to DBR as in IDBR, and sinks work as relay forward data to the base station without processing which increases the power of field nodes.

5.4. End-to-End Delay. In the case of end-to-end delay, the packet in the IDBR routing method experiences more delay than those in the DBR method. Table 5 and Figure 6 show the comparison end-to-end delay between IoT enabled UWSN and IoT based on the end-to-end delay between UWSN without IoT (DBR) and UWSN with IoT (IDBR) calculated at different periods in terms of seconds. After 1000 
TABLE 3: Stability period (alive nodes).

\begin{tabular}{|c|c|c|c|c|c|c|c|c|}
\hline \multirow{2}{*}{ Routing method } & \multicolumn{8}{|c|}{ Time (sec) } \\
\hline & 1000 & 2000 & 3000 & 4000 & 5000 & 6000 & 7000 & 8000 \\
\hline UWSN (DBR) & 195 & 169 & 149 & 137 & 131 & 107 & 105 & 104 \\
\hline UWSN-IoT (IDBR) (proposed) & 212 & 193 & 171 & 160 & 145 & 130 & 125 & 108 \\
\hline
\end{tabular}

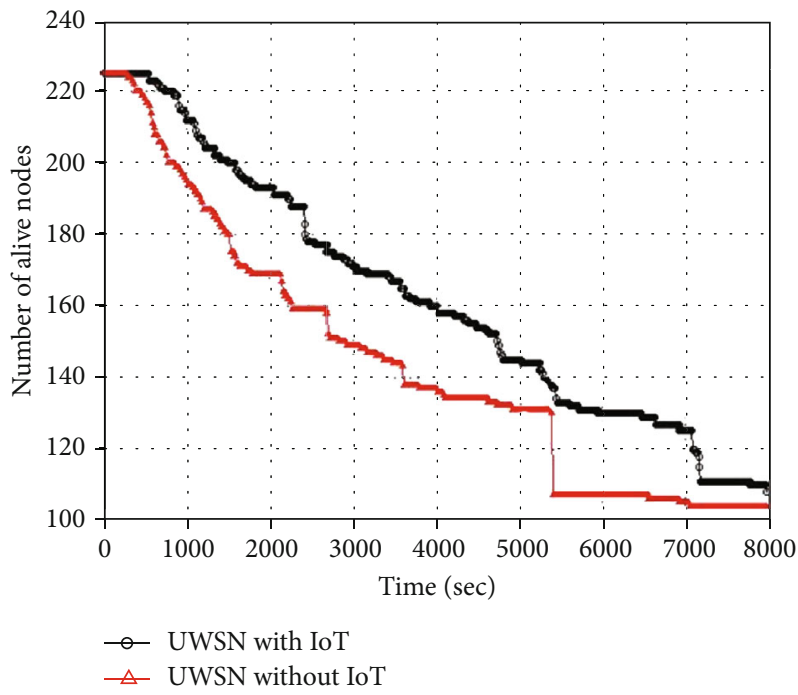

FIgURe 4: Stability period.

TABLE 4: Surface sink node utilization (dB).

\begin{tabular}{|c|c|c|c|c|c|c|c|c|}
\hline \multirow{2}{*}{ Routing method } & \multicolumn{8}{|c|}{ Time $(\mathrm{sec})$} \\
\hline & 1000 & 2000 & 3000 & 4000 & 5000 & 6000 & 7000 & 8000 \\
\hline UWSN (DBR) & 121.3 & 117.2 & 114.9 & 114.0 & 106.1 & 85.21 & 84.51 & 82.02 \\
\hline UWSN-IoT (IDBR) (proposed) & 124.8 & 127.3 & 128.3 & 133.2 & 117.6 & 114.4 & 113.5 & 100.7 \\
\hline
\end{tabular}

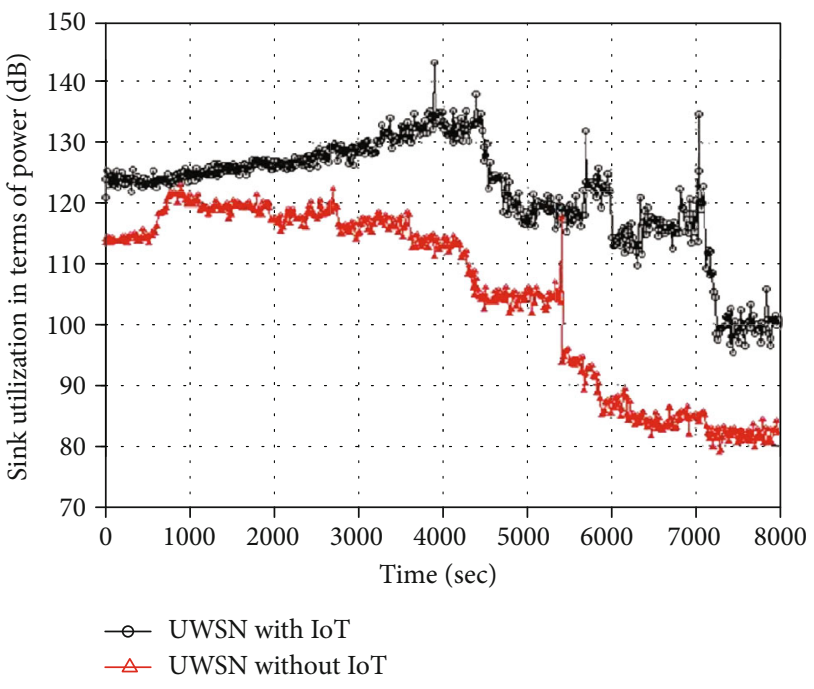

FIgURE 5: Surface sink node utilization (dB). seconds, the results showed that the end-to-end delay of packets in DBR was 30.61 seconds, whereas, in IDBR, the end-to-end delay of packets was 72.98 seconds. Similarly, after 5000 seconds, the end-to-end delay of packets in DBR was 6.46 seconds, whereas, in IDBR, the end-to-end delay of packets was 31.7 seconds. At the end of the simulation, the end-to-end delay of packets in DBR is recorded as 1.28 seconds, whereas in IDBR, the end-to-end delay of packets is recorded as 27.26 seconds. The results show that the average end-to-end delay increases IDBR compared to DBR due to the increase in distance and links between source to destination after introducing base station and cloud to enable IoT features.

\section{Conclusions}

Underwater wireless sensor network (UWSN) can be efficiently used for exploring the underwater medium for various activities such as environmental monitoring, water pollution, marine life monitoring, oil rig maintenance, and military application. However, the significant problems 
TABle 5: End-to-end delay (sec).

\begin{tabular}{|c|c|c|c|c|c|c|c|c|}
\hline \multirow{2}{*}{ Routing method } & \multicolumn{8}{|c|}{ Time (sec) } \\
\hline & 1000 & 2000 & 3000 & 4000 & 5000 & 6000 & 7000 & 8000 \\
\hline UWSN (DBR) & 30.61 & 27.96 & 24.85 & 15.28 & 6.46 & 3.55 & 2.86 & 1.28 \\
\hline UWSN-IoT (IDBR) (proposed) & 72.98 & 58.16 & 42.52 & 34.99 & 31.7 & 27.59 & 27.43 & 27.26 \\
\hline
\end{tabular}

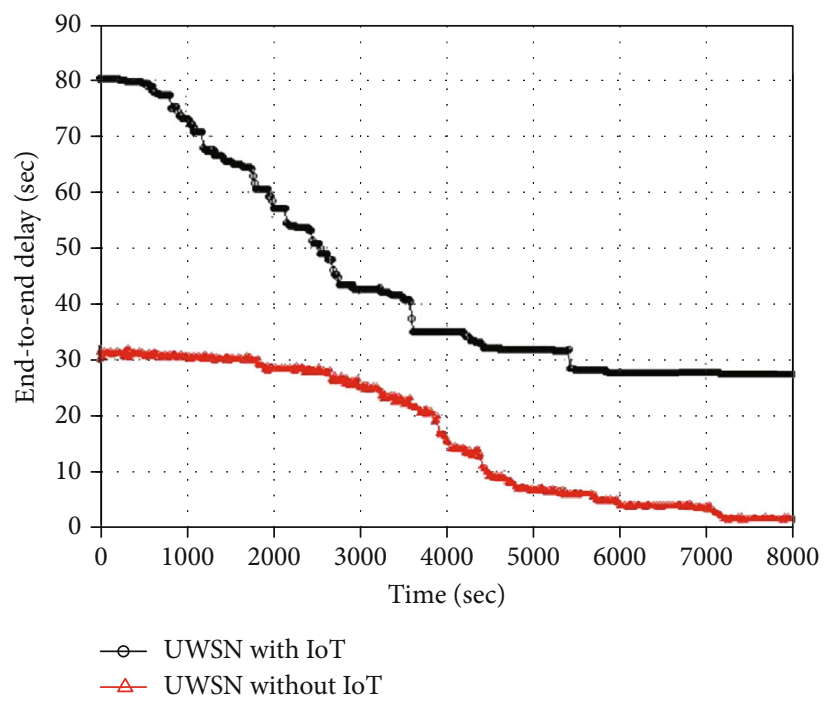

Figure 6: End-to-end delay (sec).

TABLE 6: List of abbreviations and acronyms used in this article.

\begin{tabular}{lc}
\hline Abbreviation & Explanation \\
\hline DBR & Depth base routing \\
IDBR & IoT-based depth base routing \\
UWSN & Underwater wireless sensor network \\
IoT & Internet of Things \\
MAC & Media Access Control \\
CSMA/CA & Carrier sense multiple access/collision avoidance \\
SDMA & Spatial Division Multiple Access \\
LRCLE & Location finding algorithm to reduce \\
GPS & communication cost and localization error \\
TBRL & Global Positioning System \\
(ACH $)^{2}$ & Time-based reliable link \\
& Away Cluster Head with Adaptive \\
U-(ACH) & Clustering Habit \\
SEEORVA & Underwater Away Cluster Head with \\
SOM & Adaptive Clustering Habit \\
DAI & Secure and energy-efficient opportunistic routing \\
& protocol with void avoidance \\
& Self-organizing map \\
& Distributed artificial intelligence
\end{tabular}

related to the UWSNs are limited network lifetime, the low processing capability of nodes, and high energy consumption to run routing protocols. In this research, we proposed IoT enabled Depth Base Routing method (IDBR) to utilize energy efficiently. The performance of the proposed IDBR method is compared with conventional DBR protocol using simulation conducting in MATLAB. The performance of both the methods (i.e., IDBR and DBR) is evaluated in network energy consumption, the number of alive nodes, sink utilization, and end-to-end delay.

According to the results, the proposed IDBR is found better compared to the DBR in terms of energy consumption and network stability. It was found that IDBR consumes $27.7 \%$ less energy and increases network stability time as compared to the DBR. Similarly, the utilization of the surface sinks in IDBR is more as compared to DBR as in IDBR, and sinks work as relay forward data to the base station without processing which increases the power of field nodes. The proposed mechanism improves the lifetime of the network bust and increases the accessibility and security of the sensed data. The proposed IDBR methods can effectively use environmental monitoring, ocean sampling and ecological monitoring such as the amount of chemical and biological pollution deposited on the seabed and conduct water quality observation involving people's participation affected by real-time notifications.

Optimal and efficient energy consumption is one of the vital needs of UWSN, as replacing the battery is a challenging task in the underwater environment. Our proposed IDBR method works file with the static nodes. In future, we are looking forward to testing our proposed protocol with mobile nodes, including underwater vehicles. Furthermore, we aim to develop an energy efficient routing protocol for nodes with the hybrid energy harvesting capability in UWSN. 
Table 6 includes a description of abbreviations and acronyms used in this research article.

\section{Data Availability}

It is simulation-based research, and no data were used.

\section{Conflicts of Interest}

The authors declare that there are no conflicts of interest regarding the publication of this paper.

\section{Acknowledgments}

This research was supported by Taif University Researchers Supporting Project number (TURSP-2020/254), Taif University, Taif, Saudi Arabia.

\section{References}

[1] N. Usman, O. Alfandi, S. Usman et al., "An energy efficient routing approach for IoT enabled underwater wsns in smart cities," Sensors, vol. 20, no. 15, 2020.

[2] A. Khan, I. Ali, A. Ghani et al., "Routing protocols for underwater wireless sensor networks: taxonomy, research challenges, routing strategies and future directions," Sensors, vol. 18 , no. $5,2018$.

[3] S. V. Kochergin and V. V. Fomin, "Variational identification of the inderwater pollution source power," in Processes in GeoMedia-Volume II, Springer, 2021.

[4] P. N. Mahalle, P. A. Shelar, G. R. Shinde, and N. Dey, "Introduction to underwater wireless sensor networks," in The Underwater World for Digital Data Transmission, Springer, 2021.

[5] G. Sahu and S. S. Pawar, "IOT-based underwater wireless communication," in Innovations in Computer Science and Engineering, Springer, 2021.

[6] P. Gite, A. Shrivastava, K. M. Krishna, G. H. Kusumadevi, R. Dilip, and R. M. Potdar, "Under water motion tracking and monitoring using wireless sensor network and Machine learning," Materials Today: Proceedings, 2021.

[7] E. Felemban, F. K. Shaikh, U. M. Qureshi, A. A. Sheikh, and S. B. Qaisar, "Underwater sensor network applications: a comprehensive survey," International Journal of Distributed Sensor Networks, vol. 11, no. 11, Article ID 896832, 2015.

[8] M. Jahanbakht, W. Xiang, L. Hanzo, and M. Rahimi Azghadi, "Internet of underwater things and big marine data analytics-a comprehensive survey," IEEE Communications Surveys \& Tutorials, vol. 23, no. 2, pp. 904-956, 2021.

[9] R. Ullah, A. W. Abbas, M. Ullah et al., "EEWMP: an IoT-based energy-efficient water management platform for smart irrigation," Scientific Programming, vol. 2021, Article ID 5536884, 9 pages, 2021.

[10] R. Zagrouba, A. AlAbdullatif, K. AlAjaji et al., "Authenblue: a new authentication protocol for the industrial Internet of Things," Computers, Materials \& Continua, vol. 67, no. 1, pp. 1103-1119, 2021.

[11] Z. Hussain, R. H. Shah, and N. A. Memon, "Sensor based survival detection system in earthquake disaster zones," IJCSNS, vol. 18 , no. 5,2018 .

[12] A. A. Zaidan, B. B. Zaidan, M. Y. Qahtan et al., "A survey on communication components for IoT-based technologies in smart homes," Telecommunication Systems, vol. 69, no. 1, pp. 1-25, 2018.

[13] G. Keramidas, N. Voros, and M. Hübner, Components and Services for IoT Platforms, Springer, 2016.

[14] H. Yan, Z. J. Shi, and J.-H. Cui, "DBR: depth-based routing for underwater sensor networks," in International Conference on Research in Networking, Singapore, Singapore, 2008.

[15] N.-T. Nguyen, T. T. T. le, H. H. Nguyen, and M. Voznak, "Energy-efficient clustering multi-hop routing protocol in a UWSN," Sensors, vol. 21, no. 2, 2021.

[16] M. Ammar, K. Ibrahimi, M. Jouhari, and J. Ben-Othman, "MAC protocol-based depth adjustment and splitting mechanism for underwater sensor network (UWSN)," in 2018 IEEE Global Communications Conference (GLOBECOM), Abu Dhabi, United Arab Emirates, 2018.

[17] J. Wang, J. Shen, W. Shi, G. Qiao, S. Wu, and X. Wang, "A novel energy-efficient contention-based MAC protocol used for OAUWSN," Sensors, vol. 19, no. 1, p. 183, 2019.

[18] G. Khan and R. Dwivedi, "LRCLE-location finding algorithm to reduce communication COST and localization error for acoustic sensor nodes in UWSN," International Journal on Information Technologies and Security, vol. 10, no. 4, pp. 37-44, 2018.

[19] T. Ali, M. Irfan, A. Shaf et al., "A secure communication in IoT enabled underwater and wireless sensor network for smart cities," Sensors, vol. 20, no. 15, p. 4309, 2020.

[20] V. Menon, D. Midhunchakkaravarthy, S. John, S. Jacob, and A. Mukherjee, "A secure and energy-efficient opportunistic routing protocol with void avoidance for underwater acoustic sensor networks," Turkish Journal of Electrical Engineering \& Computer Sciences, vol. 28, no. 4, pp. 2303-2315, 2020.

[21] P. Goswami, A. Mukherjee, R. Hazra et al., "AI based energy efficient routing protocol for intelligent transportation system," IEEE Transactions on Intelligent Transportation Systems, pp. 1-10, 2021

[22] P. Verma, S. Shaw, K. Mohanty, P. Richa, R. Sah, and A. Mukherjee, "A survey on hierarchical based routing protocols for wireless sensor network," in 2018 International Conference on Communication, Computing and Internet of Things (IC3IoT), Chennai, India, 2018. 